University of Wollongong

Research Online

Australian Institute for Innovative Materials -

Papers

Australian Institute for Innovative Materials

$1-1-2019$

\title{
Zinc-Tiered Synthesis of 3D Graphene for Monolithic Electrodes
}

Xiangfen Jiang

National Institute For Materials Science, Nanjing University, City University of Hong Kong

Ruiqing Li

Nanjing University

Ming $\mathrm{Hu}$

East China Normal University

Zheng $\mathrm{Hu}$

Nanjing University

Dmitri Goldberg

Queensland University of Technology, National Institute for Materials Science

See next page for additional authors

Follow this and additional works at: https://ro.uow.edu.au/aiimpapers

Part of the Engineering Commons, and the Physical Sciences and Mathematics Commons

Research Online is the open access institutional repository for the University of Wollongong. For further information contact the UOW Library: research-pubs@uow.edu.au 


\title{
Zinc-Tiered Synthesis of 3D Graphene for Monolithic Electrodes
}

\begin{abstract}
A high-surface-area conductive cellular carbon monolith is highly desired as the optimal electrode for achieving high energy, power, and lifetime in electrochemical energy storage. 3D graphene can be regarded as a first-ranking member of cellular carbons with the pore-wall thickness down to mono/fewatomic layers. Current 3D graphenes, derived from either gelation or pyrolysis routes, still suffer from low surface area, conductivity, stability, and/or yield, being subjected to methodological inadequacies including patchy assembly, wet processing, and weak controllability. Herein, a strategy of zinc-assisted solid-state pyrolysis to produce a superior 3D graphene is established. Zinc unprecedentedly impregnates and delaminates a solid ("nonhollow") char into multiple membranes, which eliminates the morphological impurities ever-present in the previous pyrolyses using solid-state carbon precursors. Zinc also catalyzes the carbonization and graphitization, and its in situ thermal extraction and recycling enables the scaled-up production. The created 3D graphene network consists integrally of morphologically and chemically pure graphene membranes. It possesses unrivaled surface area, outstanding stability, and conductivity both in air and electrolyte, exceeding preexisting 3D graphenes. The advanced 3D graphene thus equips a porous monolithic electrode with unparalleled energy density, power density, and lifetime in electric-double-layer capacitive devices.
\end{abstract}

\section{Disciplines}

Engineering | Physical Sciences and Mathematics

\section{Publication Details}

Jiang, X., Li, R., Hu, M., Hu, Z., Goldberg, D., Bando, Y. \& Wang, X. (2019). Zinc-Tiered Synthesis of 3D Graphene for Monolithic Electrodes. Advanced Materials, 31 (25), 1901186-1-1901186-9.

\section{Authors}

Xiangfen Jiang, Ruiqing Li, Ming Hu, Zheng Hu, Dmitri Goldberg, Yoshio Bando, and Xuebin Wang 


\section{Zinc-Tiered Synthesis of 3D Graphene for Monolithic Electrodes}

Xiang-Fen Jiang, Ruiqing Li, Ming Hu, Zheng Hu, Dmitri Golberg, Yoshio Bando, and Xue-Bin

Wang*

Dr. X.-F. Jiang, Dr. R. Q. Li, Prof. X.-B. Wang National Laboratory of Solid State

Microstructures Collaborative Innovation Center of Advanced Microstructures College of

Engineering and Applied Sciences

Nanjing University Nanjing 210093, China

E-mail: wangxb@nju.edu.cn,wangxb@fuji.waseda.jp

Dr. X.-F. Jiang, Prof. D. Golberg, Prof. Y. Bando

WPI Center for Materials Nanoarchitectonics (MANA) National Institute for Materials

Science (NIMS) Tsukuba 3050044, Japan

Dr. X.-F. Jiang

Department of Materials Science and Engineering City University of Hong Kong

Hong Kong 999077, China

Prof. M. Hu

State Key Laboratory of Precision Spectroscopy School of Physics and Materials Science

East China Normal University Shanghai 200241, China

Prof. Z. Hu

Key Laboratory of Mesoscopic Chemistry of Ministry of Education School of Chemistry

and Chemical Engineering

Nanjing University Nanjing 210093, China

Prof. D. Golberg

School of Chemistry, Physics and Mechanical Engineering Queensland University of

Technology

Brisbane, QLD 4000, Australia

Prof. Y. Bando

Institute of Molecular Plus Tianjin University

Tianjin 300072, China

Prof. Y. Bando

Australian Institute for Innovative Materials University of Wollongong

North Wollongong, NSW 2500, Australia

A high-surface-area conductive cellular carbon monolith is highly desired as the optimal electrode for achieving high energy, power, and lifetime in electrochemical energy storage. 3D graphene can be regarded as a first-ranking member of cellular carbons with the pore-wall thickness down to mono/few-atomic layers. Current 3D graphenes, derived from either gelation or pyrolysis routes, still suffer from low surface area, conductivity, stability, and/or yield, being subjected to methodological inadequacies including patchy assembly, wet processing, and weak controllability. Herein, a strategy of zinc-assisted solid-state pyrolysis to produce a superior 3D graphene is established. Zinc unprecedentedly impregnates and delaminates a solid ("nonhollow") char into multiple membranes, which eliminates the morphological impurities ever-present in the previous 
pyrolyses using solid-state carbon precursors. Zinc also catalyzes the carbonization and graphitization, and its in situ thermal extraction and recycling enables the scaled-up production. The created 3D graphene network consists integrally of morphologically and chemically pure graphene membranes. It possesses unrivaled surface area, outstanding stability, and conductivity both in air and electrolyte, exceeding preexisting 3D graphenes. The advanced 3D graphene thus equips a porous monolithic electrode with unparalleled energy density, power density, and lifetime in electric-double-layer capacitive devices. 
Three characteristics (energy, power, and lifetime) and cost-performance ratio (CPR) are the core for the wide implementation of an energy system. Electrochemical capacitors present high power yet poor energy density. High cost per energy unit is the main bottleneck of growing market of supercapacitors. 3D network structure can improve energy and power of electrochemical electrodes (including nonFaradaic or Faradaic ones for supercapacitors/batteries) ${ }^{[1]} \mathrm{A}$ cellular carbon monolith is envisaged as a candidate of the best 3D-network electrodes, owing to the lightweight, conductivity, stability, and opulence of carbon. However, the present porous carbon monoliths have the limited specific surface area (SSA). Activated carbon has high SSA, but it is a powder and the micropore-dominated SSA is electrochemically inaccessible. Powders must be bound into monoliths, yet binders deteriorate energy, power, and stability of electrodes. A high-SSA conductive stable carbon monolith is highly desired yet remains challenging for supercapacitors. The ultimate version of porous carbons, when the framework thins as monoatomiclayer, can in principle achieve large SSA, which is just conceptually 3D

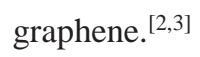

Graphene is favored in electrode materials for large surface and extraordinary electron mobility attributive to $2 \mathrm{D}$ crystals. ${ }^{[4,5]}$ In practice, the intrinsic graphenic advantages are severely suppressed by irreversible face-to-face restacking and deficient hand-in-hand interconnection in a macroscopic aggregate of graphene powders. ${ }^{[6,7]} 3 \mathrm{D}$ graphene is conceived as a porous monolithic architecture composed of single/few-layered graphenes, which plays a crucial role to deliver the nanoscaled advantages of individuals to macroscopic bulks. Theoretical prediction indicated that 3D graphene can realize unprecedented surface, mechanical, and electronic characteristics. ${ }^{[8]}$ Therefore, 3D graphene is recognized as an advanced material for vast potentials, e.g., as electrodes, fillers, adsorbents, and elastomers for electrochemical energy storage, electrocatalysis, thermal management, sorption, separation, and mechanics.

The fabrication of 3D graphenes has been intensively pursued for the past nine years, yet the reported products suffer from either unsatisfying quality and/or low throughput. ${ }^{[8,9]}$ In general, three ways were developed (see more in Table S1 in the Supporting Information). i) With the primary concept of solutionbased assembly, reduced graphene oxide (RGO) platelets were noncovalently built into 3DRGO. It employed gelation-related technologies including bulky gelation, ${ }^{[10,11]}$ interfacial gelation, ${ }^{[12]}$ freezecasting, ${ }^{[13]}$ and additive-assisted gelation/pore forming. ${ }^{[14]}$ However, the weak joints in bridging RGO platelets break the continuous transport of electrons and phonons. Plenty of oxygen impurities and intrinsic defects in RGO degrade the stability. ${ }^{[15,16]}$ The aqueous processing presents a dilemma: the drying of hydrous products causes agglomeration and cracks while the remedial lyophilization is timeand energy- consuming. The RGO production yet brings forth a huge amount of wastewater. ii) Instead of patchwork assembling, growing 3D graphenes via pyrolyzing organics devoted better structural integrity. In a nontemplating growth, the foaming and pyrolysis of sugars yielded a high-throughput 3D strutted graphene, ${ }^{[17]}$ but its surface area is diminished by solid ("nonhollow") carbon struts, and it is hard to control the structural geometry. iii) On the other hand, a templating growth was more predictable. Chemical vapor deposition (CVD) generated graphene foams as the positive hollow replica of porous monolithic templates, e.g., nickel foams. ${ }^{[18]}$ Nevertheless, the throughput is low, being afflicted by the tedious processing and complex cycling of parent templates. Alternatively, pyrolysis of solid-state/fluidic carbon sources with powdery templates cast a negative replica identified as 3D carbon-graphene, ${ }^{[19,20]}$ since heavy solid carbons coexist also as morphological impurities. The wet etching is necessary to 
remove the templates in both cases. It injures surface-attached delicate graphenes, increases cost, and inflicts the aqueous processing problem again. In short, current prototypes of 3D graphenes still cannot meet the expectation. The large-scale production of a high-quality 3D graphene remains a great challenge.

Herein we establish a strategy of zinc-assisted solid-state pyrolysis (ZASP) to tackle the challenge. Metal zinc introduces a tiering process (a new carbon-metal interaction) beyond the normal templating. The tiering effect guides the delamination of bulky organics or solid chars, which thus removes the intrinsic problem, i.e., solid morphological impurity formed in pyrolyses employing solid-state/fluidic precursors. Evaporative zinc additionally spurs the carbonization-graphitization of organics, precludes the troublesome wet processing, and realizes the in situ easy regeneration of metal agents. ZASP thus enables a high-quality high-throughput 3D graphene toward ideal porous monolithic electrodes for low-CPR electrochemical devices.

We compacted the powders of glucose and zinc (typically 1:9) into the glucose-zinc ingot, and then heated it to straightforwardly produce a monolith of all-membrane well-graphitized 3D graphene (Figure 1a,b). We name the product as Zn-guided 3D graphene (ZNG) for the specific roles played by zinc. The production has a high throughput, and the product preserves the designed appearance for targeted applications, e.g., $0.5 \mathrm{~g}$ specimen in Figure 1b,c.

ZNG is a 3D continuous network, geometrically consisting of polyhedral cells in intimate arrangement (Figure 1d; Figure S1a, Supporting Information). Each cell has 5-6 neighbors on a section, tending to close-packed configuration. In terms of foam geometry, face, edge, and vertex are defined as the meeting regions of two, three, and four polyhedral cells, respectively. Three faces meet as an edge, and four edges converge into a vertex. The edge/vertex elements of ZNG are played by a wavy bundle of gauzy membranes, and the face is served by the extended membrane (Figure 1e-g; Figure S1b,c, Supporting Information). ZNG does not have any morphological impurity, in contrast with the reported 3D graphenes/carbons derived from solid-state/fluidic carbon sources which had morphological impurities such as solid struts, particles, etc. ${ }^{[17,19]}$

The membranes of ZNG are mono/few-atomic layers with the mean thickness as small as $2.2 \mathrm{~nm}$ (Figure 1h). Raman spectrum of ZNG presents a clear 2D peak as compared to 3DRGO (Figure 1i). G peak of ZNG locates at $1580 \mathrm{~cm}^{-1}$, while $\mathrm{G}$ band of 3DRGO is blueshifted by $17 \mathrm{~cm}^{-1}$, because of $\mathrm{D}^{\prime}$ peak activated by abundant defects of RGO. The uniformity of graphene membranes is further examined by Raman mapping (Figure S1d, Supporting Information). Briefly, the atomic ordering of the membranes prevails over popular RGO (Figure S1e, Supporting Information) and freely pyrolyzed graphenic sheets, ${ }^{[17]}$ although they are not as good as CVD or cleavage derived graphenes.

The better in-plane periodicity and graphitization degree of membranes of ZNG are confirmed by X-ray diffraction (XRD) in Figure S2a,b (Supporting Information). Electron energy-loss spectroscopy (EELS) analysis in Figure S2c (Supporting Information) reveals the high fraction of $\mathrm{sp}^{2}$-hybridized carbons (as high as 98\%) and tiny elemental impurities (with only oxygen of 1.0 at $\%$ ), better than other 3D graphenes (see Table S2 in the Supporting Information). The result echoes as the little amount of oxygenic groups in Fourier transform infrared spectroscopy (FTIR) in Figure S2d (Supporting Information). 
The structural and crystalline integrity of ZNG brings high stability, conductivity, and robust mechanics. The onset oxidation temperature of ZNG is $613^{\circ} \mathrm{C}$ in air (Figure 2a), higher than those of 3DRGO (447 ${ }^{\circ} \mathrm{C}$ ) and activated carbon $\left(532{ }^{\circ} \mathrm{C}\right)$. Resistivity is more sensitive to oxidation. Conductance of ZNG deteriorates since $530{ }^{\circ} \mathrm{C}$ (Figure $2 \mathrm{~b}$ ), which is highly elevated as compared with $3 \mathrm{DRGO}\left(380{ }^{\circ} \mathrm{C}\right)$. Both results verify the high stability of ZNG. Next, the conductivity of ZNG monolith is determined as $1.6 \mathrm{~S}$ $\mathrm{cm}^{-1}$ at room temperature, higher than 3DRGO $\left(0.22 \mathrm{~S} \mathrm{~cm}^{-1}\right)$ and compressed strutted graphenes $(0.5 \mathrm{~S}$ $\mathrm{cm}^{-1}$ ) at similar density. It stems from the negligible impurity and well graphitization degree of membranes, and especially from the 3D continuous network of ZNG. The conductivity of ZNG is comparatively temperature-invariant (Figure S3a, Supporting Information). On the contrary, the conductivity of 3DRGO varies strongly and irreversibly following temperature. In addition, ZNG is elastic and compressible for the 3D microstructure (Figure S3b, Supporting Information). The membrane structure of ZNG contributes high slenderness (ratio of cell size to membrane thickness), which results in reversible local bending for generating macroscopic elasticity. ZNG can recover after compression with a low energy loss coefficient of 34\%. In contrast, the intersheet sliding and de-binding in 3DRGO leads to a high energy loss of $67-87 \%$, and the fragile failure in traditional carbon foams gives poor reversibility. ${ }^{[3,13]}$

To verify the availability and regulation of ZASP route, we control the production by adjusting the dimension of zinc microspheres and the feed ratio of glucose to zinc. The topological structures of ZNG can be reliably customized by tuning the size of zinc (Figure 2c; Figure S4a-c, Supporting Information). Larger cells are accordingly yielded if using larger zinc microspheres. With altering the glucose feeding in 5-20 wt \%, SSA of ZNG is documented up to $2020 \mathrm{~m}^{2} \mathrm{~g}^{-1}$. The high SSA is contributed mainly by the external surface and the pores with the width of $>2 \mathrm{~nm}$ (Figure 2d). All "pores" are rather open as indicated by the small hysteresis, coinciding with microscopic observation. SSA of all ZNG products are quite high (2020-1390 $\left.\mathrm{m}^{2} \mathrm{~g}^{-1}\right)$, as confirmed by the small membrane thicknesses averaging at 2.0-3.0 $\mathrm{nm}$ (Figure 2e,f). The SSA is top-ranked in the porous carbon family (e.g., typically $1000-2000 \mathrm{~m}^{2} \mathrm{~g}^{-1}$ for activated carbon), which benefits from the intersupported all-membrane configuration preventing the restacking. Especially, it is better than other types of 3D graphenes: 3DRGO $\left(<1000 \mathrm{~m}^{2} \mathrm{~g}^{-1}\right),{ }^{[21-23]}$ strutted graphenes $\left(1005 \mathrm{~m}^{2} \mathrm{~g}^{-1}\right),{ }^{[17]}$ and CVD-derived graphene foams $\left(850 \mathrm{~m}^{2} \mathrm{~g}^{-1}\right)^{[18]}$ (see Table S2 in the Supporting Information).

Three merits further illuminate the feasibility and robustness of ZASP for practical mass production. i) Zinc microspheres volatilize during the pyrosynthesis and are concurrently regenerated in an exhaust system, facile for reusing to produce ZNG (Figure S4d-f, Supporting Information). Zinc is the only nonhazardous volatile metal among the thirteen metals with a boiling point of $<1000{ }^{\circ} \mathrm{C}(\mathrm{Na}, \mathrm{K}, \mathrm{Rb}, \mathrm{Cs}$, Fr, Bk, Cf, As, Te, Po, Hg, Cd, and $\mathrm{Zn}$ ). The in situ recycling of zinc reduces the cost and prompts the scalability. ZNG costs only $\approx 1$ US dollar $\mathrm{g}^{-1}$ in our lab trial, lower than CVD-derived graphene foams and 3DRGO (see the note of Table S1 in the Supporting Information). ii) The vaporization of zinc simplifies the route free from troublesome wet treatment, evidently beneficial to upgrading the quality of products. Zinc can be completely evaporated, leaving little residue around $0.01 \mathrm{wt} \%$. iii) The solidstate carbon source makes the route exempt from the less efficient gaseous feedstocks, ${ }^{[24]}$ and the zinc tiering effect proposed hereinafter eliminates the intrinsic obstacle of impurity in pyrolyses of solidstate/fluidic carbon sources. Solid-state precursors further enable the arbitrary shaping, compatible with 
established casting or emerging 3D printing. The solid-state synthesis thus represents a breakthrough toward industrialization production of 3D graphenes.

To explore the growth mechanism, we first examined the influence of zinc on the chemical evolution of glucose in four stages (Figures S5-S10, Supporting Information). i) Zinc partially reacts with the steam from glucose to generate $\mathrm{ZnO}$, as revealed by the depression of water signal around $200{ }^{\circ} \mathrm{C}$ in online TGFTIR in Figure S6d (Supporting Information) and by the $\mathrm{ZnO}$ appeared in FTIR in Figure S8a (Supporting Information). ii) At $150-400{ }^{\circ} \mathrm{C}$, zinc and $\mathrm{ZnO}$ catalyze the caramelization, as evidenced by the preceding mass loss of glucose-zinc (see the A-position in Figure $3 \mathrm{a}$ and Figure S5c in the Supporting Information). The catalysis is confirmed by the control glucose- $\mathrm{ZnO}$ (Figure $3 \mathrm{a}$ ) and the more released furfural from glucose-zinc (Figure S6d, Supporting Information). iii) At $400-1000{ }^{\circ} \mathrm{C}$, zinc species accelerate the carbonization with elimination of heteroatoms, including decomposition of hydrogenated carbons (see isothermal TG of the model char in Figure S7 in the Supporting Information) and oxygenated carbons (Figures S5c, S6d, and S10, Supporting Information). The catalysis appears clearly in the control glucose- $\mathrm{ZnO}$ (the B-position in Figure $3 \mathrm{a}$ and Figure S5c in the Supporting Information). The $\mathrm{ZnO}$ (it is quantitatively much less than zinc) liberates oxygen via carbothermal reduction over $750{ }^{\circ} \mathrm{C}$ (Figures S6d and S8a, Supporting Information). iv) Above $1000{ }^{\circ} \mathrm{C}$, the graphitization is promoted by zinc, as reflected by isothermally annealing another model char in Figure S9 (Supporting Information). It might be attributed to liquid-carbon interfacial catalytic graphitization ${ }^{[25]}$ and thickness-dependent graphitization easy for delaminated chars. ${ }^{[26]}$ These evidences unveil a picture of zinc catalytic fast pyrolysis (CFP) upon glucose (Figure S10, Supporting Information). CFP offers a barrier-reduced carbonization-graphitization for higher graphitization degree.

More attention is paid to probing the role of zinc in guiding morphological evolution in two stages. i) Zinc impregnates into char at lower temperature (Figure 3f,g). During the synthesis, glucose-derived char can adsorb and ensnare zinc vapor, as is evident from the mass gain over $480{ }^{\circ} \mathrm{C}$ in the control $\mathrm{TG}$ of pure glucose built in zinc vapor (the $C$-position in Figure $3 \mathrm{a}$ and Figure S5c in the Supporting Information). The process is confirmed by the surface energy test of the model char films (Figure S11, Supporting Information), where the low-temperature char can be wetted by nanosized zinc droplets. We consider that low-temperature char carries plenty of heteroatoms and $\mathrm{sp}^{2}$-layer edges, so zinc would interact with the disordered carbon and the mesophase char in style of either Lewis acid or base. Considering the abundance of zinc (mass ratio of zinc to char is 50:1 at $500{ }^{\circ} \mathrm{C}$ ), the char can be fully impregnated with zinc. Accordingly, a miscible char-zinc intermediate appears in microscopes in Figure $3 \mathrm{~b}$ and Figure S12 (Supporting Information). ii) Zinc tiers a solid char into several lamellae at higher temperature (Figure $3 \mathrm{~h}$ ). Along with heating to $800{ }^{\circ} \mathrm{C}$, the embedded zinc is gradually extruded from the char-zinc matrix, which orientates the lamellar texture, as observed in Figure $3 \mathrm{c}-\mathrm{e}$. The extrusion is believed to be driven by the diminishing surface energy of the char with eliminating heteroatoms and merging edges, as well as the coarsening zinc (govern by Ostwald-Freundlich or Kelvin equation), as evidenced in Figure S11 (Supporting Information). The lamellar texture might rely on anisotropic $\mathrm{sp}^{2}$ carbon layers having minimal surface energy. After the complete vaporization of zinc, it turns into a graphene aerogel (Figure 3i).

The adsorption, segregation, delamination, and extraction of zinc in/out of chars is collectively proposed as the tiering effect. It loosens and transforms a solid char into several atomically thin graphitic 
membranes. The tiering process is robust and efficient, as implicated by high SSA of ZNG. It resembles the disintegration phenomenon (soaking and cavitation) of coke bricks by zinc in blast furnace. The tiering effect distinguishes zinc from other metals and oxides. 3D counterparts templated by calcium, scandium, titanium, vanadium, chromium, manganese, iron, cobalt, nickel, and copper are plagued by morphological impurities and wet postetching, as experimented in Figures S13 (Supporting Information) and sketched in Figure 3j-m. Glucose gathers in the spaces of edges and vertices in raw workpieces, which finally becomes thick solid carbons such as struts, shells, pellets, etc. ${ }^{[2,3,17,19]}$ The tiering effect also does not occur when directly evaporating copper instead of wet etching. Cadmium guides a similar 3D graphene, stressing the tiering effect of zinc family. Such a unique effect is the key to weed out morphological impurity for graphene-only structure.

To evaluate the surface, stability, and conductivity of porous ZNG in electrolyte, we studied the ZNG electrode in a non-Faradaic process. ZNG shows spacious electrochemically accessible surface area, with an electric double layer (EDL) capacitance of $336 \mathrm{~F} \mathrm{~g}^{-1}$ at $0.5 \mathrm{~A} \mathrm{~g} \mathrm{~g}^{-1}$. The capacitance is higher than those of 3DRGO and other carbons, as learnt from the larger steady-state current in cyclic voltammetry (CV) in Figure 4a. It results from the high SSA. In addition, the nearly ideal capacitive behaviors are preserved at high speeds for the 3D network structure (Figures 4b; Figure S14, Supporting Information).

ZNG electrodes present high electrochemical stability. ZNG has a flat slope of $0.2 \mathrm{mS}$ at $0.2-0.8 \mathrm{~V}$ in $\mathrm{CV}$ in Figure $4 \mathrm{a}$, much lower than $0.5 \mathrm{mS}$ for 3DRGO, showing the electrochemical inertness. Corrosiveresistant $Z N G$ is further supported by equivalent parallel resistance (EPR) via chronoamperometry. EPR of ZNG is $21 \mathrm{k} \Omega$. In contrast, EPR of 3DRGO is $17 \mathrm{k} \Omega$ and it has pseudocapacitive lump in CV. Furthermore, numerous galvanostatic cycling of ZNG electrodes demonstrate the high stability against Faradaic corrosion (Figure 4c). ZNG electrodes work for 267000 cycles at a raised voltage of $1.4 \mathrm{~V}$, and the life achieves 1300000 cycles at a rated voltage of $1.0 \mathrm{~V}$ (using an acceleration factor of 5 , see the Experimental Section, Supporting Information). ${ }^{[27]}$

ZNG electrodes display small internal resistance (IR), as implied by the steep slopes around 0 or $1 \mathrm{~V}$ in CV in Figure 4a. Galvanostatic discharging measures direct-current (DC) IR. When switching charging to discharging programs, the action time (in millisecond or microsecond) is neglected by an electrochemical workstation. We develop a temporally continuous monitoring in the chargingdischarging. A quick-stepping signal generator (Solartron 1280B) supplies the switching, and a hightemporal-resolution monitor (VersaSTAT 4) independently records the terminal voltage. Such data are gapless in the time axis, as shown in Figure 4d,e. We further develop an accurate DC analysis about the voltage response of porous electrodes to the current step, to get IR and to factorize IR (Figure S15, Supporting Information). IR includes the ionic resistance in pores, the equivalent series resistance (ESR, namely the Ohmic resistance), and the pseudoresistance contributed by the self-discharge. The smaller IR of ZNG electrodes is dominated by the smaller ESR, as compared to 3DRGO (Figure 4f). DCESR is 0.11 and $0.58 \Omega$ for ZNG and 3DRGO electrodes, respectively (as supported by alternate-current AC analysis in Figure S16 in the Supporting Information). Consequently, the in-electrolyte resistance of the solid-state network of ZNG is $\approx 5$ times lower than that of 3DRGO, in line with their air-ambient resistivities, as summarized in Figure S15 and Table S3 (Supporting Information). The result verifies that the high conductance of ZNG keeps up in electrolyte. 
With revealing the three splendid properties of ZNG substantially retained in the electrochemical environment, ZNG behaves like ideal porous electrodes. The intersupported configuration of the all membrane structure offers the maximally exposed electrochemical surface area. The high chemical stability ensures the electrochemical longevity. The interconnected channel facilitates the smooth in-pore ion flow. The 3D continuous graphene network enables the rapid electron transport along the framework.

ZNG is deservedly applied to electrochemical capacitors. ${ }^{[28]}$ ZNG electrodes present the highest specific capacitance (336 $\mathrm{F} \mathrm{g}^{-1}$ ) among EDL-type capacitors. The capacitances of pure and nearly pure carbon materials appeared within $250 \mathrm{~F} \mathrm{~g}^{-1}$ for moderate SSA $\left(\approx 1000 \mathrm{~m}^{2} \mathrm{~g}^{-1}\right),{ }^{[17,29-33]}$ and was limited within 300 $\mathrm{F} \mathrm{g}^{-1}$ for high SSA $\left(\approx 2000 \mathrm{~m}^{2} \mathrm{~g}^{-1}\right) \cdot{ }^{[16,34-36]}$ Our ZNG-based EDL is actually a significant progress approaching the upper limit. Three-electrode test can generate higher data, which are not used herein. Ultramicropore might show desolvation-enhanced capacitance with a compromise on lowering power. ${ }^{[37,38]}$ Some "carbons" were reported with high capacitances owing to involved pseudocapacity. For instance, $1-6$ at $\%$ nitrogen doping pulls up the capacitance by $50 \%,{ }^{[34,39-42]}$ and 10 at $\%$ oxygen doping adds $\approx 40 \%$ capacitance (the numbers got via surveying tens of literatures for qualitative reference). The Faradaic reactive heteroatoms are active yet less stable, like that observed with pseudocapacitive oxides and polymers. ${ }^{[43]}$ Electrochemical windows is reduced because of heteroatom-catalyzed redox such as $\mathrm{H}_{2} / \mathrm{O}_{2}$ evolutions, ${ }^{[44]}$ which is detrimental to life of supercapacitors. EDL mode can survive much longer than the pseudocapacitive behavior, so ZNG electrodes demonstrate the longest life (Table S4, Supporting Information).

The ZNG-based supercapacitors achieve the top-ranked energy density and maximum power density, 11.7 $\mathrm{Wh} \mathrm{kg}^{-1}$ and $625 \mathrm{~kW} \mathrm{~kg}^{-1}$ in $\mathrm{H}_{2} \mathrm{SO}_{4}$ system, excelling 3DRGO and other 3D graphenes (Figure $4 \mathrm{~g}$ h; Table S4, Supporting Information). They are further improved in 1-ethyl-3-methylimidazolium tetrafluoroborate $\left(\mathrm{EMIMBF}_{4}\right.$ ), being 179 and $1800 \mathrm{~kW} \mathrm{~kg}$, respectively (Figure S18, Supporting Information). The volumetric energy density is estimated as $60 \mathrm{Wh} \mathrm{L}^{-1}$ under encapsulation pressure. For 10 year term devices, the life-cycle assessment is valuable for accounting the total cost. ${ }^{[45]}$ Lifecycle stored energy of ZNG-based supercapacitors reaches $15 \mathrm{MWh} \mathrm{kg}^{-1}$, undoubtedly prevailing over other 3D graphenes' counterparts, pseudocapacitors, and commercial devices (Figure 4i). The regenerationfree ZNG electrodes can reduce the cost per energy unit. It frees CPR from the need for lifecycle assessment, which boosts ZNG-based supercapacitors toward practical applications.

In summary, the zinc-introduced tiering effect features the ZASP route, creating the all-membrane structure. It makes ZASP stand out from normal pyrolyses and other synthetic protocols. Zinc is also a chemical accelerant offering well graphitization, and it is a thermoremovable agent capable of in situ recyclability. ZASP is robust, reliable, and controllable, and it is a dry-media approach exempt from wet processing and gaseous feedstock. The bottom-up production can be easily scaled up with the compatible industrial establishments in powder metallurgy, sintering, and investment casting.

All the advantages deliver a high-throughput ZNG with comprehensively superior chemical purity, stability, conductivity, and SSA. It fundamentally suffices for architectural design and integration and opens a path to industrial production. The ZNG foam is further applied as an advanced binder-free porous monolithic electrode as a sampling of immense potentials. Its supercapacitors supply the high energy density, power density, lifetime, and life-cycle stored energy, outperforming state-ofthe-art energy 
devices. The progress facilitates the widespread implementation of ZNG-based pristine and hybrid electrodes toward diverse cells/batteries. It has not escaped our notice that the cost-effective ZNG opens up a wide horizon to push forward the practice of 3D graphenes for electrodes, sorbents, composite fillers, bioscaffolds, elastomers, and auxetics.

Experimental Section

Growth procedures, characterizations, and tests are presented in the Supporting Information.

Supporting Information

Supporting Information is available from the Wiley Online Library or from the author.

Acknowledgements

The authors are grateful to Profs. Rodney S. Ruoff, Tetsuya Osaka, Kazuyuki Kuroda, Haoshen Zhou, Zhong-Lin Wang, Jie Tang, and Qinghong Yuan for valuable discussions, and thank Drs. Min Zhou, Benjamin Cunning, and Masanori Mitome for the helps. The authors acknowledge the support of 1000-Talents Program, National Natural Science Foundation of China (NSFC 51672124, 21603096), Natural Science Foundation of Jiangsu Province of China (BK20160618), Fundamental Research Funds for the Central Universities (021314380144), and Technical Center of Nano Fabrication and Characterization, Laboratory of Solid State Microstructure, Nanjing University.

Conflict of Interest

A relevant patent application is in progress on the basis of the work.

Keywords

3D graphene foam, porous monolithic electrode, solid-state precursor, synthesis

[1] M. F. El-Kady, Y. L. Shao, R. B. Kaner, Nat. Rev. Mater. 2016, 1, 16033.

[2] A. A. Zakhidov, R. H. Baughman, Z. Iqbal, C. X. Cui, I. Khayrullin, S. O. Dantas, J. Marti, V. G. Ralchenko, Science 1998, 282, 897.

[3] M. Inagaki, J. S. Qiu, Q. G. Guo, Carbon 2015, 87, 128.

[4] F. Bonaccorso, L. Colombo, G. H. Yu, M. Stoller, V. Tozzini, A. C. Ferrari, R. S. Ruoff, V. Pellegrini, Science 2015, 347 , 1246501 .

[5] K. S. Novoselov, V. I. Falko, L. Colombo, P. R. Gellert, M. G. Schwab, K. Kim, Nature 2012, 490, 192.

[6] M. R. Lukatskaya, B. Dunn, Y. Gogotsi, Nat. Commun. 2016, 7, 12647.

[7] Y. X. Xu, G. Q. Shi, X. F. Duan, Acc. Chem. Res. 2015, 48, 1666.

[8] A. Dasgupta, L. P. Rajukumar, C. Rotella, Y. Lei, M. Terrones, Nano Today 2017, 12, 116

[9] K. Shehzad, Y. Xu, C. Gao, X. F. Duan, Chem. Soc. Rev. 2016, 45, 5541.

[10] D. Li, M. B. Muller, S. Gilje, R. B. Kaner, G. G. Wallace, Nat. Nanotechnol. 2008, 3, 101.

[11] Y. X. Xu, K. X. Sheng, C. Li, G. Q. Shi, ACS Nano 2010, 4, 4324

[12] X. W. Yang, L. Qiu, C. Cheng, Y. Z. Wu, Z. F. Ma, D. Li, Angew. Chem., Int. Ed. 2011, $50,7325$.

[13] L. Qiu, J. Z. Liu, S. L. Y. Chang, Y. Z. Wu, D. Li, Nat. Commun. 2012, 3, 1241.

[14] X. D. Huang, K. Qian, J. Yang, J. Zhang, L. Li, C. Z. Yu, D. Y. Zhao, Adv. Mater. 2012, 24, 4419.

[15] A. Bagri, C. Mattevi, M. Acik, Y. J. Chabal, M. Chhowalla, V. B. Shenoy, Nat. Chem. 2010, $2,581$.

[16] Y. W. Zhu, S. Murali, M. D. Stoller, K. J. Ganesh, W. W. Cai, P. J. Ferreira, A. Pirkle, R. M. Wallace, K. A. Cychosz, M. Thommes, D. Su, E. A. Stach, R. S. Ruoff, Science 2011, 332, 1537.

[17] X. B. Wang, Y. J. Zhang, C. Y. Zhi, X. Wang, D. M. Tang, Y. B. Xu, Q. H. Weng, X. F. Jiang, M. Mitome, D. Golberg, Y. Bando, Nat. Commun. 2013, 4, 2905.

[18] Z. P. Chen, W. C. Ren, L. B. Gao, B. L. Liu, S. F. Pei, H. M. Cheng, Nat. Mater. 2011, 10, 424.

[19] J. W. Sha, C. T. Gao, S. K. Lee, Y. L. Li, N. Q. Zhao, J. M. Tour, ACS Nano 2016, 10, 1411. 
[20] J. Zhao, Y. F. Jiang, H. Fan, M. Liu, O. Zhuo, X. Z. Wang, Q. Wu, L. J. Yang, Y. W. Ma, Z. Hu, Adv. Mater. $2017,29,1604569$.

[21] H. T. Sun, L. Mei, J. F. Liang, Z. P. Zhao, C. Lee, H. L. Fei, M. N. Ding, J. Lau, M. F. Li, C. Wang, X. Xu, G. L. Hao, B. Papandrea, I. Shakir, B. Dunn, Y. Huang, X. F. Duan, Science 2017, 356, 599.

[22] Y. X. Xu, C. Y. Chen, Z. P. Zhao, Z. Y. Lin, C. Lee, X. Xu, C. Wang, Y. Huang, M. I. Shakir, X. F. Duan, Nano Lett. 2015, 15, 4605.

[23] Y. X. Xu, Z. Y. Lin, X. Zhong, X. Q. Huang, N. O. Weiss, Y. Huang, X. F. Duan, Nat. Commun. 2014, 5, 4554.

[24] Z. Z. Sun, Z. Yan, J. Yao, E. Beitler, Y. Zhu, J. M. Tour, Nature 2010, 468, 549.

[25] J. I. Fujita, R. Ueki, Y. Miyazawa, T. Ichihashi, J. Vac. Sci. Technol., B: Microelectron. Nanometer Struct.-Process., Meas., Phenom. 2009, 27, 3063.

[26] H. Hatori, Y. Yamada, M. Shiraishi, Carbon 1993, 31, 1307.

[27] O. Bohlen, J. Kowal, D. U. Sauer, J. Power Sources 2007, 172, 468.

[28] P. Simon, Y. Gogotsi, B. Dunn, Science 2014, 343, 1210.

[29] J. J. Yoo, K. Balakrishnan, J. S. Huang, V. Meunier, B. G. Sumpter, A. Srivastava, M. Conway, A. L. M. Reddy, J. Yu, R. Vajtai, P. M. Ajayan, Nano Lett. 2011, 11, 1423.

[30] Y. X. Xu, Z. Y. Lin, X. Q. Huang, Y. Liu, Y. Huang, X. F. Duan, ACS Nano 2013, 7, 4042.

[31] X. W. Yang, J. W. Zhu, L. Qiu, D. Li, Adv. Mater. 2011, 23, 2833.

[32] X. W. Yang, C. Cheng, Y. F. Wang, L. Qiu, D. Li, Science 2013, 341, 534.

[33] P. Pachfule, D. Shinde, M. Majumder, Q. Xu, Nat. Chem. 2016, 8, 718.

[34] T. Q. Lin, I. W. Chen, F. X. Liu, C. Y. Yang, H. Bi, F. F. Xu, F. Q. Huang, Science 2015, 350, 1508.

[35] K. Xie, X. T. Qin, X. Z. Wang, Y. N. Wang, H. S. Tao, Q. Wu, L. J. Yang, Z. Hu, Adv. Mater. 2012, $24,347$.

[36] M. F. El-Kady, V. Strong, S. Dubin, R. B. Kaner, Science 2012, 335, 1326.

[37] J. Chmiola, G. Yushin, Y. Gogotsi, C. Portet, P. Simon, P. L. Taberna, Science 2006, 313, 1760.

[38] M. Salanne, B. Rotenberg, K. Naoi, K. Kaneko, P. L. Taberna, C. P. Grey, B. Dunn, P. Simon, Nat. Energy 2016, 1, 16070.

[39] W. L. Zhang, C. Xu, C. Q. Ma, G. X. Li, Y. Z. Wang, K. Y. Zhang, F. Li, C. Liu, H. M. Cheng, Y. W. Du, N. J. Tang, W. C. Ren, Adv. Mater.

2017, 29, 1701677

[40] J. Xu, Z. Q. Tan, W. C. Zeng, G. X. Chen, S. L. Wu, Y. Zhao, K. Ni, Z. C. Tao, M. Ikram, H. X. Ji, Y. W. Zhu, Adv. Mater. 2016, 28,5222 .

[41] P. Chen, J. J. Yang, S. S. Li, Z. Wang, T. Y. Xiao, Y. H. Qian, S. H. Yu, Nano Energy 2013, 2, 249.

[42] L. L. Zhang, X. Zhao, H. X. Ji, M. D. Stoller, L. F. Lai, S. Murali, S. Mcdonnell, B. Cleveger, R. M. Wallace, R. S. Ruoff, Energy Environ. Sci. 2012, 5, 9618.

[43] Y. L. Shao, M. F. El-Kady, C. W. Lin, G. Z. Zhu, K. L. Marsh, J. Y. Hwang, Q. H. Zhang, Y. G. Li, H. Z. Wang, R. B. Kaner, Adv. Mater. 2016, 28, 6719.

[44] J. T. Zhang, Z. H. Zhao, Z. H. Xia, L. M. Dai, Nat. Nanotechnol. 2015, 10, 444.

[45] B. Zakeri, S. Syri, Renewable Sustainable Energy Rev. 2015, 42, 569. 

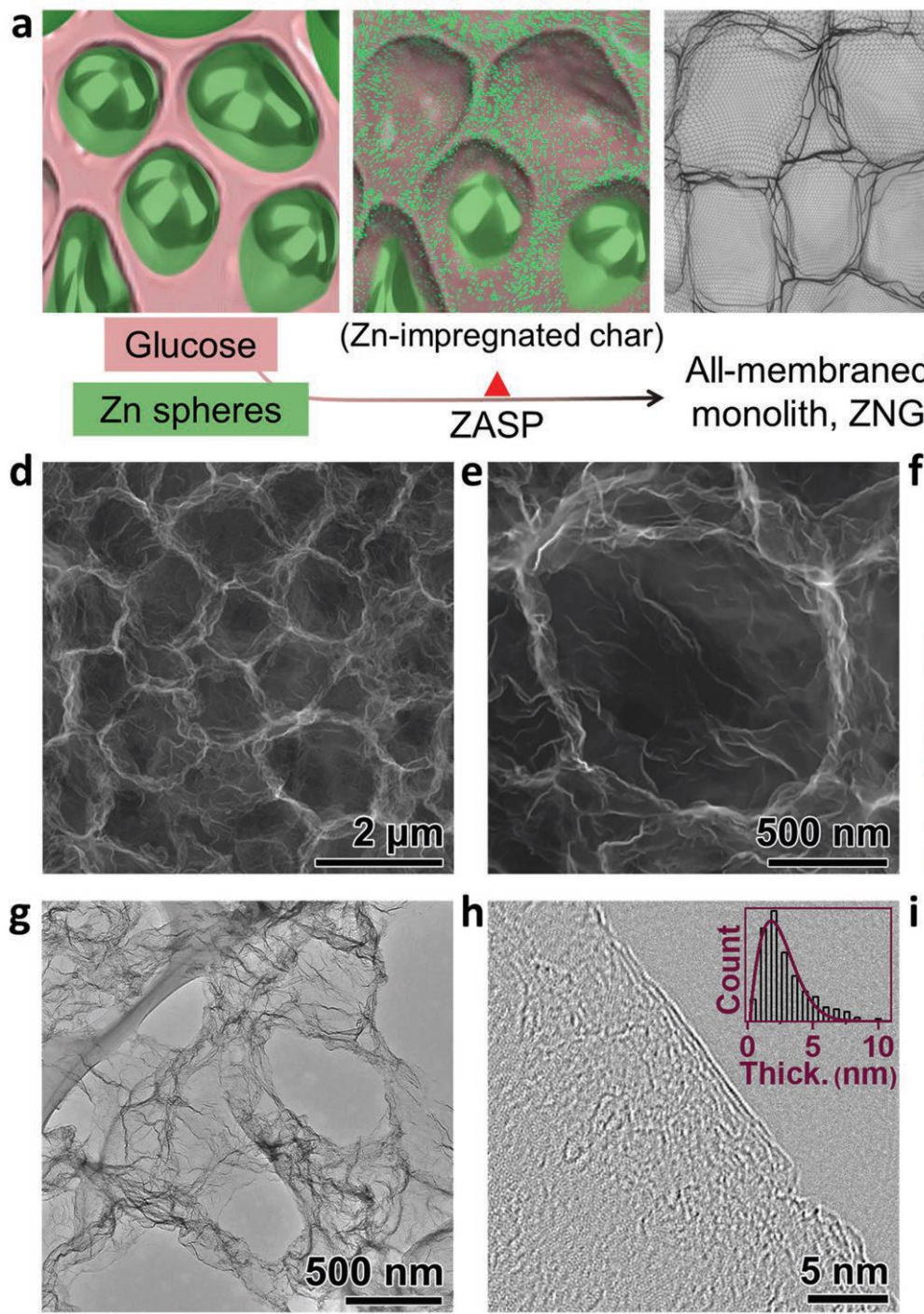

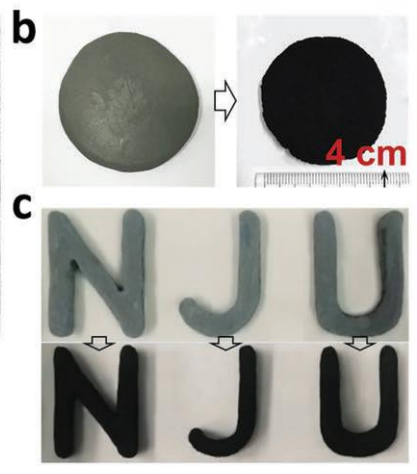
All-membraned

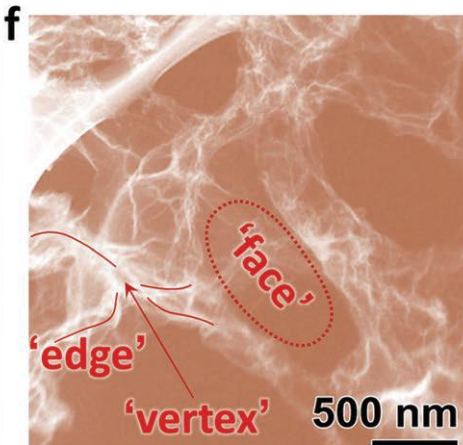

i

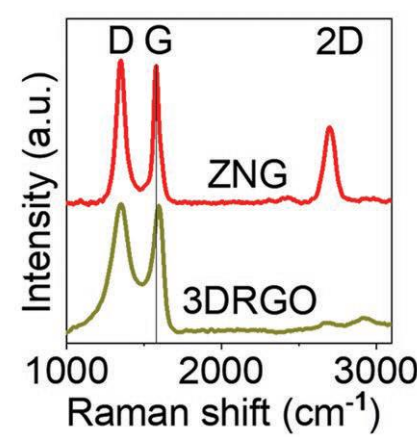

Figure 1. Synthetic protocol, structure, morphology, and spectroscopic analyses of ZNG. a) Scheme of ZASP route undergoing a char-zinc intermediate. $b, c)$ Photos of preshaped glucose-zinc workpiece and corresponding ZNG with a density of $34 \mathrm{mg} \mathrm{cm}^{-3}$. d) Scanning electron microscopy (SEM) image of ZNG. e-g) SEM, dark-field scanning transmission electron microscopy (STEM), and transmission electron microscopy (TEM) images of ZNG cells. Not only the face but also the edge/vertex consist exclusively of morphologically pure graphene membranes. $h$ ) High-resolution TEM (HRTEM) image of an individual graphene membrane with 1-3 atomic layers. Inset is statistics of membrane thickness with a Weibull distribution curve. i) Raman spectra of ZNG versus 3DRGO. 
a

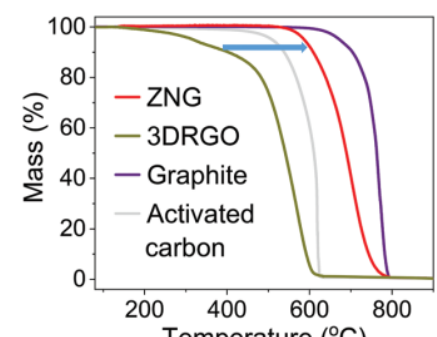

d

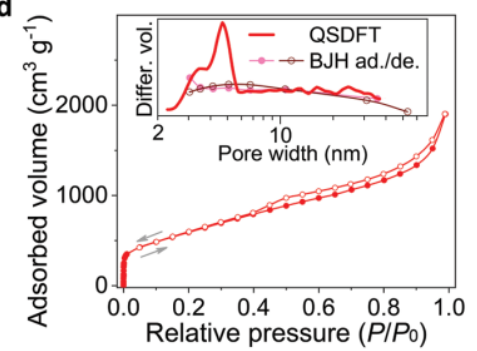

b
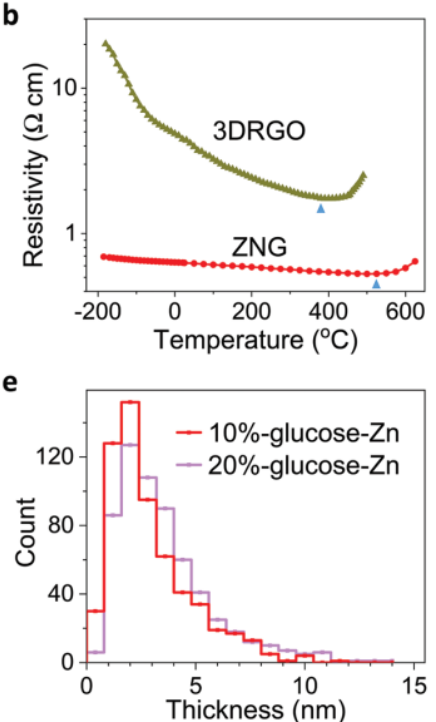
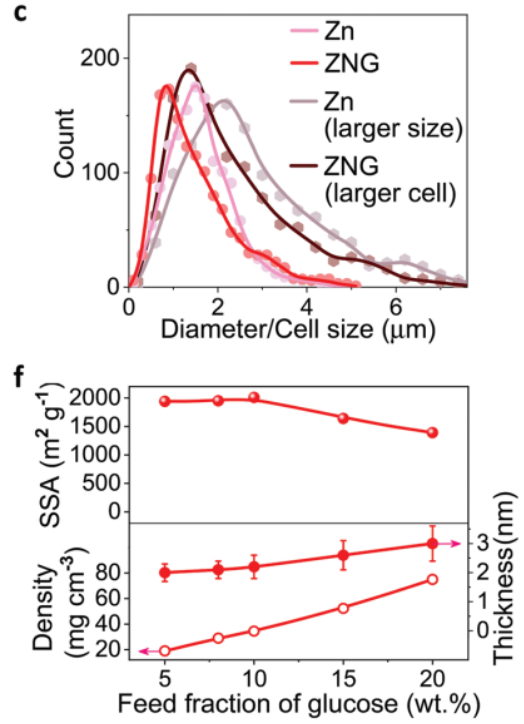

Figure 2. Stability, conductivity, SSA, and regulation of ZNG. a) Thermogravimetry (TG) of ZNG and references in air.

b) Resistivity of ZNG versus 3DRGO following temperature in air. c) Statistics of diameters of two zinc microspheres (means are 1.6 and $2.6 \mu \mathrm{m}$ ) and cell sizes of corresponding ZNG (1.3 and $2.0 \mu \mathrm{m}$ ) via SEM observation. d) Nitrogen adsorption-desorption isotherms of ZNG. The inset shows pore size distributions based on quenched solid density function theory (QSDFT) and Barrett-Joyner-Halenda (BJH) method. e) Thickness statistics via TEM observation upon membranes of ZNG obtained at the marked feeding. f) Regulation of SSA, membrane thickness, and density of ZNG. The density varies almost linearly correlating with volumetric proportion. 


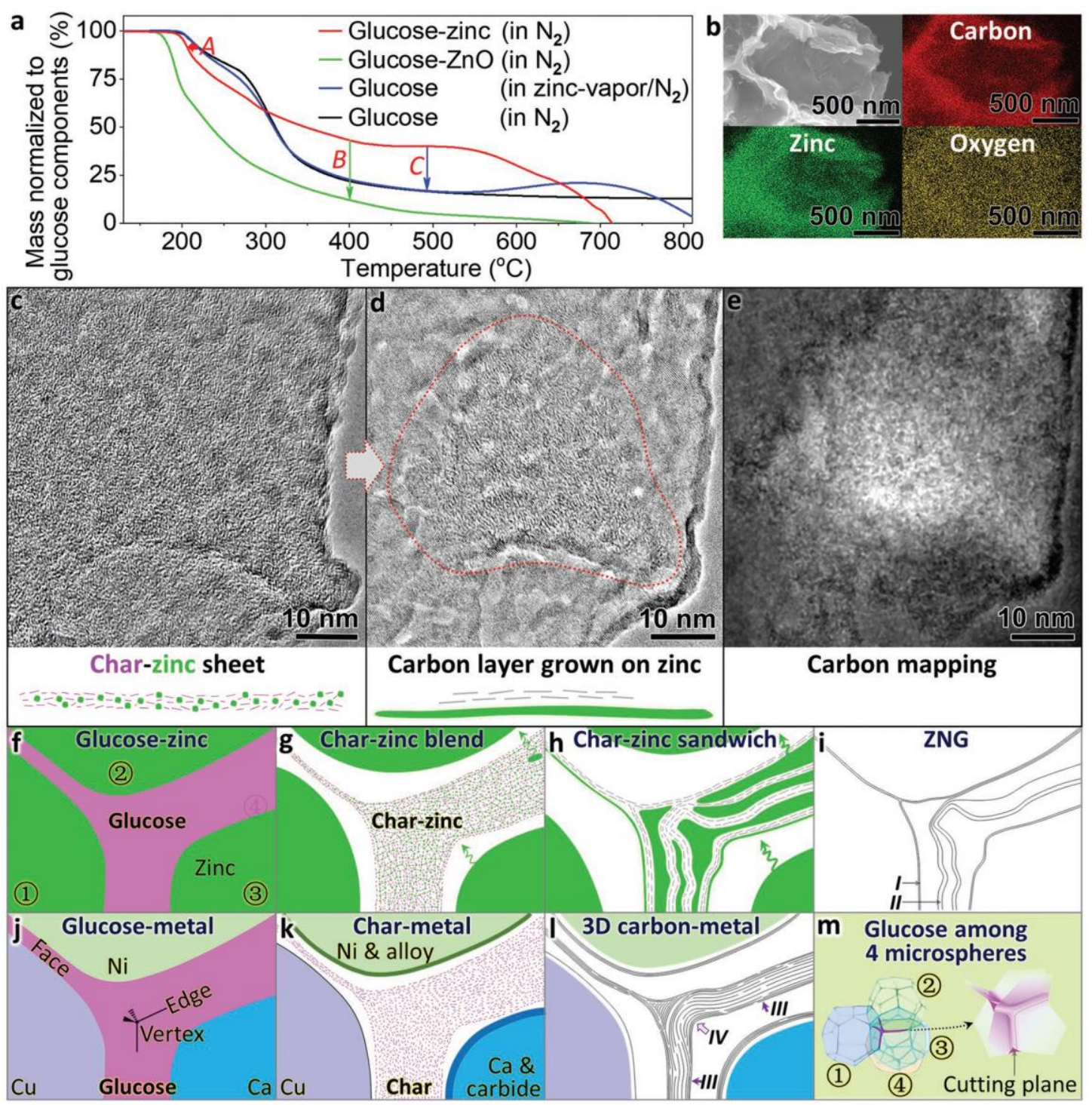

Figure 3. Proposed mechanism of zinc prompted pyrolysis and zinc tiering effect. a) TG of glucose-zinc in nitrogen, two references (glucose and glucose- $\mathrm{ZnO}$ ) in nitrogen, and the third reference (glucose) in a flow of nitrogen-carried zinc vapor. b) SEM and elemental mapping of a cell of char-zinc intermediate taken at $700{ }^{\circ} \mathrm{C}$ in synthesis. c) TEM image and sketch of a char-zinc sheet (the face of cells) taken at $700{ }^{\circ} \mathrm{C}$. It was more homogeneous at the first glance. d) Resultant carbon film in situ growing on zinc background obtained by irradiating (c) under an electron beam, which mimics the segregation between zinc and char. e) EELS mapping for identifying carbon. f) Sectional diagram of glucose passing 1 face, 2 edges, and 1 vertex among 4 zinc microspheres. g) Zinc impregnates char via dynamic evaporationcondensation in heating. h) Zinc delaminates char into $\pi-\pi$ stacked fragment, accompanying catalytic carbonization and zinc coarsening. i) Char lamellae become graphene membranes, so that the edge/vertex is a loose bundle of membranes. I and // denote vertical and tilted double-layered graphenes, respectively. j-I) Pyrolysis of glucose among nontiering metals, yielding inevitable nonmembrane morphological impurities (III and IV mark the solid graphitic strut and tetrapod joint. m) Geometry of model of glucose syrup among four metal microspheres that are drawn as polyhedrons for showing connective regions ( 6 faces, 4 edges, and 1 vertex). 

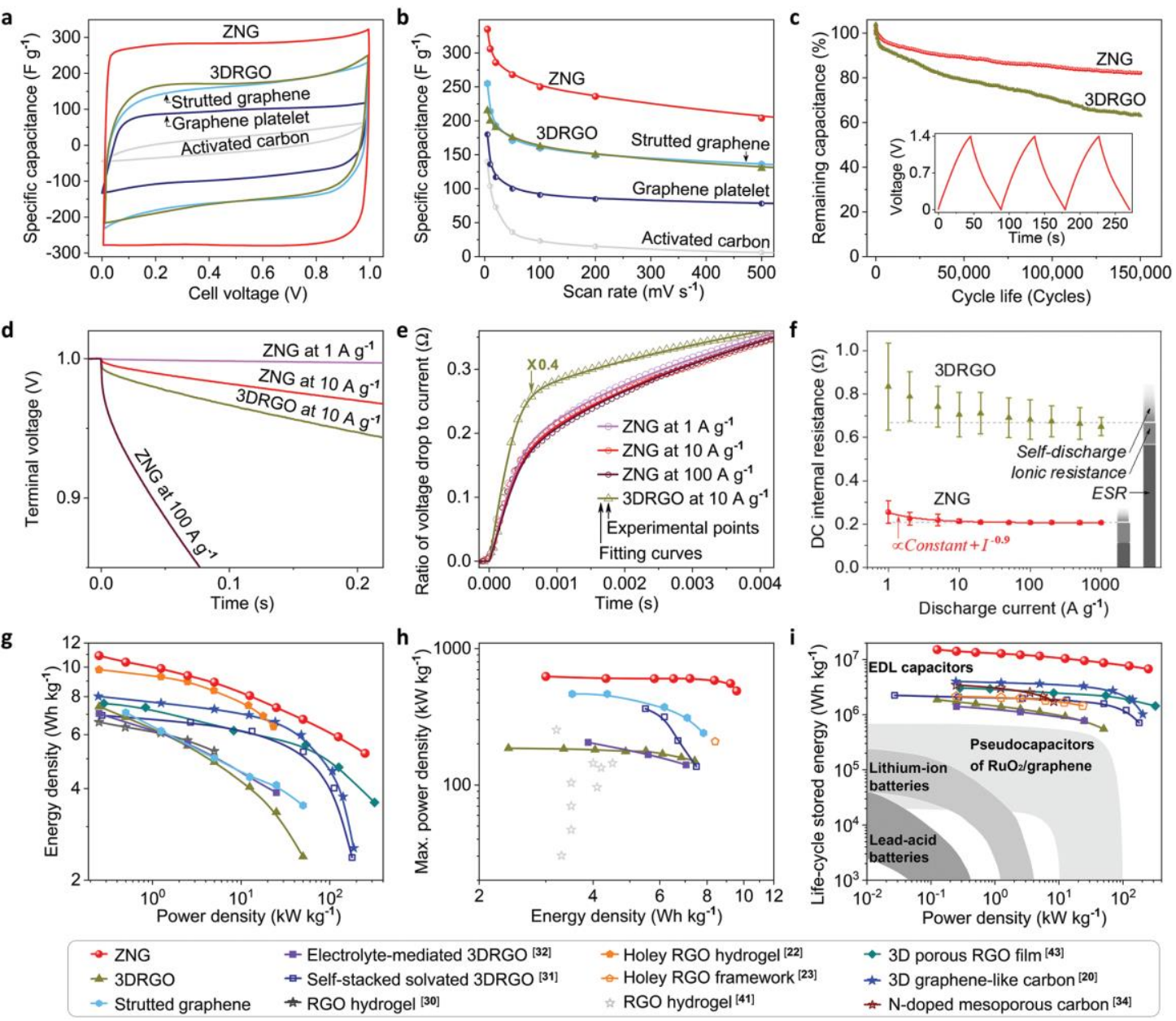

$\leftarrow 3 \mathrm{D}$ porous $\mathrm{RGO}$ film ${ }^{[43]}$

* 3D graphene-like carbon [20]

* N-doped mesoporous carbon ${ }^{[34]}$

Figure 4. Electrochemical properties of ZNG and comparison of ZNG-based supercapacitors with state-of-the-art devices. a) CV of symmetric electrodes of ZNG and references at a scan rate of $100 \mathrm{mV} \mathrm{s}^{-1}$ in $1 \mathrm{~m} \mathrm{H}_{2} \mathrm{SO}_{4}$. b) Correlation between capacitance and scan rate. c) Accelerated power cycling of ZNG and 3DRGO electrodes at $1.4 \mathrm{~V}$ at room temperature. Inset is galvanostatic charging-discharging at $5 \mathrm{~A} \mathrm{~g}^{-1}$. The capacitance undergoes initial increase owing to pseudocapacitive activation and developing permeation, exponential fading owing to deactivation of pseudocapacity, and final linear decay owing to corrosion. d) Terminal voltage of ZNG electrodes following time, as measured by the temporally continuous monitoring in potentiostatic charging and galvanostatic discharging. e) Ratio of the voltage drop to the discharging current, i.e., apparent resistances converted from (d). The curves are fitted according to Equation (S12) in the Supporting Information, which are independent of currents. f) IR and its component. IR difference is dominated by ESR, while in-pore ionic resistances are similar and self-discharge contributes variable residues. g) Ragone chart of aqueous supercapacitors based on ZNG, 3DRGO, compressed strutted graphene, and others. h) Tradeoff chart of maximum power density versus energy density. i) Life-cycle stored energy of ZNG-based supercapacitors. 